Introduction VEGF has been implicated in the development of IPF. Alternative splicing of the VEGF-A gene generates numerous isoforms. The differential effects of these isoforms, in particular the VEGF- $\mathrm{A}_{\mathrm{xxx}} \mathrm{b}$ family, thought to have several opposing functions to the conventional family of isoforms (VEGF-A $A_{x x x} a$ ), have not been considered.

Hypothesis

- The balance of VEGF-A $A_{x x x} a: V E G F-A_{x x x} b$ isoform expression is important in the pathogenesis of IPF.

- VEGF- $\mathrm{A}_{\mathrm{xxx}} \mathrm{b}$ isoforms may be protective against the formation of pulmonary fibrosis (PF).

Methods Normal and IPF lung lysates $(\mathrm{n}=5)$ were analysed by western blotting (WB), and ELISA using an antibodies specific for PanVEGF-A and VEGF- $\mathrm{A}_{\mathrm{xxx}} \mathrm{b}$ isoforms.

The Bleomycin (BLM)-induced model of PF was used in conjunction with two transgenic (TG) mouse models, developed to explore the role of ATII-derived VEGF in the development of PF: 1) a conditionally inducible, ATII-specific, VEGF knock-out mouse (STCLL mice) and 2) a TG mouse over-expressing VEGF-A $\mathrm{A}_{\mathrm{xxx}} \mathrm{b}$ in ATII cells (MMTV-VEGF $\left.{ }_{165} \mathrm{~b}\right)$.

To explore the therapeutic potential of VEGF- $\mathrm{A}_{\mathrm{xxx}} \mathrm{b}$ in PF, wild-type mice were administered intraperitoneal (IP) injections of VEGF- $\mathrm{A}_{165} \mathrm{~b}$, commencing 10 days after BLM challenge.

In all experiments fibrosis was assessed histologically using Masson's Trichrome, with blinded scoring of tissue sections. Results By WB $(n=3)$ and ELISA $(n=5)$ there was no significant difference in PanVEGF-A expression between normal and IPF lung homogenates ( $t$-test, $\mathrm{p}>0.05)$. In contrast, VEGF$\mathrm{A}_{\mathrm{xxx}} \mathrm{b}$ expression was significantly increased in these same IPF samples compared to control, by ELISA $(t$-test, **** $<0.0001)$ and WB (Densitometry: $t$-test, ${ }^{*} \mathrm{p}<0.05$ ).

Specific deletion of VEGF-A from ATII cells of mice ameliorated the development of BLM-induced pulmonary fibrosis $(\mathrm{n}=$ 5, Lung fibrosis score: ANOVA with Holm's Sidak *p < 0.01). Over-expression of VEGF-A $\mathrm{xxx}_{\mathrm{xx}} \mathrm{b}$ in ATII cells also ameliorated the development of pulmonary fibrosis $(\mathrm{n}=6$, Lung fibrosis score: ANOVA with Holm's Sidak ***p < 0.001). Furthermore, delivery of VEGF- $\mathrm{A}_{165} \mathrm{~b}$, specifically during the fibrotic phase of the BLM model, also attenuated lung fibrosis development $(\mathrm{n}=$ 6, Lung fibrosis score: ANOVA with Holm's Sidak *p $<0.05$ ). Conclusion Changes in the bioavailability of ATII cell-derived VEGF-A, namely the ratio of VEGF-A ${ }_{x x x} a: V E G F-A_{x x x} b$, appear critical to the development of pulmonary fibrosis. This data suggests that more a targeted approach to anti-VEGF-A therapy in IPF should be explored.

\section{S65 THE ROLE OF SRC KINASE IN INSPIRATORY RESISTIVE BREATHING-INDUCED PULMONARY INFLAMMATION}

'D Toumpanakis, ${ }^{1} \mathrm{P}$ Zacharatos, ${ }^{1} \mathrm{~T}$ Michailidou, ${ }^{2} \mathrm{G}$ Tsoukalas, ${ }^{1} \mathrm{~T}$ Vassilakopoulos. ${ }^{1} 1 s t$ Department of Critical Care and Pulmonary Services, Medical School, University of Athens, Evangelismos Hospital, Athens, Greece; ${ }^{2}$ 4th Pulmonary Department, Sotiria General and Chest Diseases Hospital, Athens, Greece

\subsection{6/thoraxjnl-2015-207770.71}

Introduction and objectives Inspiratory resistive breathing (IRB), a hallmark of obstructive pulmonary diseases, is characterised by large negative intrathoracic pressures. IRB is shown to induce pulmonary inflammation in previously healthy rats. Src is a multifunctional kinase that is activated by phosphorylation upon mechanical stress and plays a significant role in inflammatory processes. The aim of our study was to investigate the role of Src in IRB-induced pulmonary inflammation.
Methods Anaesthetised, tracheostomised rats were breathing spontaneously through a 2-way non rebreathing valve. The inspiratory port was connected to a resistance, setting peak tidal tracheal pressure at $50 \%$ of maximum (IRB). Quietly breathing animals served as controls. After $6 \mathrm{~h}$ of IRB, the mechanics of the respiratory system were assessed with the forced oscillation technique. Bronchoalveolar lavage (BAL) was performed to measure total and differential cell count and total protein levels. Phosphorylation of Src and ERK was detected in lung tissue samples by Western blot analysis at $30 \mathrm{~min}, 3$ and $6 \mathrm{~h}$ of IRB. The Src inhibitor PP2 was administered intraperitoneally $(1 \mathrm{mg} /$ $\mathrm{kg}$ ), 30 min prior to IRB, in a subgroup of animals.

Results After $6 \mathrm{~h}$ of IRB, increased tissue elasticity was measured, compared to control. Increased BAL cellularity was also found (2-fold increase to control), due to raised numbers of both macrophages and neutrophils. Total protein levels were elevated in BAL fluid. Src activation was detected at $30 \mathrm{~min}$ of IRB (3-fold increase to control), while ERK was phosphorylated at 3 and $6 \mathrm{~h}$. Inhibition of Src kinase attenuated the increase in tissue elasticity after $6 \mathrm{~h}$ of IRB. Following inhibition of Src kinase, the total cell number after $6 \mathrm{~h}$ of IRB was not increased compared to control. Neither macrophage nor neutrophil count was elevated after $6 \mathrm{~h}$ of IRB, following Src inhibition. Total protein levels were not altered by Src inhibition. Src inhibition attenuated the activation of ERK only at $3 \mathrm{~h}$ of IRB.

Conclusion Src kinase activation partly mediates IRB-induced pulmonary inflammation.

\section{S66 CAFFEINE INHIBITS TGF $\beta$ ACTIVATION BY EPITHELIAL CELLS, INTERRUPTS FIBROBLAST RESPONSES TO TGF $\beta$, AND REDUCES PULMONARY FIBROSIS IN EX VIVO PRECISION-CUT LUNG SLICES}

${ }^{1}$ AL Tatler, ${ }^{2} \mathrm{~J}$ Barnes, ${ }^{1} \mathrm{~A}$ Habgood, ${ }^{1} \mathrm{~A}$ Goodwin, ${ }^{2} \mathrm{R}$ McAnulty, ${ }^{1} \mathrm{RG}$ Jenkins. ${ }^{1}$ University of Nottingham, Nottingham, UK; ${ }^{2}$ University College London, London, UK

10.1136/thoraxjnl-2015-207770.72

Caffeine (1, 3, 7-tri-methylxanthine) is a common food additive found naturally in many products. It is a non-selective competitive antagonist of G-protein coupled adenosine receptors and can inhibit phosphodiesterases. Caffeine has anti-fibrotic effects in the liver and increased caffeine consumption has been associated with reduced liver fibrosis in patients with chronic hepatitis $\mathrm{C}$ infection. The effect of caffeine on pulmonary fibrosis has not been investigated, however, it has been shown to inhibit TGF $\beta$ induced Smad signalling in epithelial cells. This study aimed to investigate the anti-fibrotic effects of caffeine in the lung using lung epithelial cells, fibroblasts and an ex vivo precision-cut lung slice (PCLS) model of fibrosis.

Immortalised human bronchial epithelial cells (iHBECs) and primary human lung fibroblasts from were used. TGF $\beta$ activation was assessed using an in vitro TGF $\beta$ reporter cell assay and assessment of phosphorylated Smad2. Expression of pro-fibrotic genes was assessed by quantitative polymerase chain reaction. Proliferation of fibroblasts was assessed by brdU incorporation assay. Finally, the effect of caffeine on established lung fibrosis was investigated ex vivo using PCLS. Mice were instilled with saline or 60 IU bleomycin and PCLS obtained after 28 days. PCLS were treated with increasing concentrations of caffeine for five days prior to measurement of collagen by high-performance liquid chromatography. Viability of the PCLS following caffeine treatment was assessed by MTT assay. 
Caffeine induced a concentration-dependent decrease in TGF $\beta$ activation in iHBECs but had no effect on TGF $\beta$ activation in lung fibroblasts. Furthermore, caffeine reduced expression of the TGF $\beta$-inducible genes PAI1 and Col1A and reduced TGFB1 transcript in epithelial cells. Additionally, caffeine reduced TGF $\beta$-induced proliferation of lung fibroblasts and reduced expression of pro-fibrotic genes including COL1A and ACTA2. Crucially, ex vivo treatment of fibrotic PCLS from bleomycin treated animals with caffeine caused a dose-dependent reduction in collagen deposition after five days. Caffeine had no effect on collagen deposition in PCLS isolated from saline treated animals nor did caffeine affect tissue viability in PCLS from either saline or bleomycin treated animals.

In conclusion, caffeine has anti-fibrotic effects in the lung via concomitant inhibition of epithelial TGF activation and fibroblast responses to $\mathrm{TGFb}$.

\section{\begin{tabular}{|l|l}
\hline S67 & VITAMIN D DEFICIENCY DRIVES PULMONARY
\end{tabular} INFLAMMATION IN A HUMAN MODEL OF THE ACUTE RESPIRATORY DISTRESS SYNDROME INDUCED BY INHALED LIPOPOLYSACCHARIDE IN HEALTHY VOLUNTEERS}

${ }^{1} \mathrm{M}$ Fitzgerald, ${ }^{1} \mathrm{M}$ Shyamsundar, ${ }^{2} \mathrm{JJ} \mathrm{MCN}$ amee, ${ }^{3} \mathrm{DR}$ Thickett, ${ }^{1} \mathrm{CM}$ O'Kane, ${ }^{1} \mathrm{DF}$ McAuley. ${ }^{1}$ Centre for Infection and Immunity, Queen's University Belfast, Belfast, Northern Ireland, UK; ${ }^{2}$ Regional Intensive Care Unit, Royal Victoria Hospital, Belfast, Northern Ireland, UK; ${ }^{3}$ Centre for Translational Inflammation Research, University of Birmingham Laboratories, Birmingham, UK

\subsection{6/thoraxjnl-2015-207770.73}

The acute respiratory distress syndrome (ARDS) is characterised by exaggerated alveolar inflammation. Vitamin D deficiency in an LPS induced murine model of ARDS results in exaggerated alveolar inflammation. However the role of vitamin D deficiency in pulmonary inflammation in humans is unclear. We hypothesised that in healthy volunteers with vitamin D deficiency, pulmonary inflammation would be increased following LPS inhalation. Methods Healthy volunteers inhaled 50 micrograms of LPS and six hours later underwent bronchoalveolar lavage for measurement of cytokines. Plasma was collected at baseline and one day post LPS inhalation for measurement of vitamin D.

Results 28 participants were included. The mean age of volunteers was $26.2+/-5.5$ years. All 28 patients were vitamin D deficient (plasma levels $<50 \mathrm{nmol} / \mathrm{l}$ ), with $89 \%$ (25/28) patients having severe vitamin D deficiency $(<25 \mathrm{nmol} / \mathrm{l})$. Vitamin D levels were significantly higher after LPS inhalation $(\mathrm{p}<0.002)$. Levels of IL$1 \beta$ in BALF were significantly higher in those with severe deficiency than those with mild/moderate deficiency (Figure 1; $\mathrm{p}=0.04$ ). Levels of IL-6, IL-8 or TNF- $\alpha$ did not differ between groups.

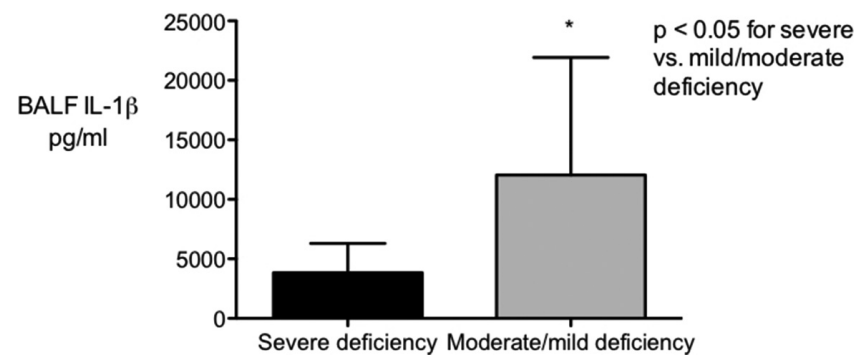

Abstract S67 Figure 1 Bronchoalveolar lavage fluid (BALF) levels of IL-1 beta were significantly elevated in volunteers with severe plasma vitamin D deficiency $(<25 \mathrm{nmol} / /)$ compared to those with mild or moderate deficiency $(25-50 \mathrm{nmol} / \mathrm{l})$
Conclusions Vitamin D deficiency was highly prevalent in this population of healthy volunteers. The rise in vitamin D levels post LPS exposure may represent mobilisation of vitamin D from fat stores during inflammation though vitamin D metabolism and kinetics are complex and may differ in healthy volunteers and the critically ill. Severe deficiency correlated with increased alveolar inflammation.

\section{Lung infection and primary ciliary dyskinesia}

\section{S68 A LONGITUDINAL STUDY CHARACTERISING A LARGE ADULT PRIMARY CILIARY DYSKINESIA COHORT}

${ }^{1} A$ Shah, ${ }^{2} A$ Shoemark, ${ }^{1} S J$ Macneill, ${ }^{2} B$ Bhaludin, ${ }^{2} A$ Rogers, ${ }^{2} D$ Bilton, ${ }^{2}$ DM Hansell, ${ }^{2} \mathrm{R}$ Wilson, ${ }^{2} \mathrm{MR}$ Loebinger. ${ }^{1} / \mathrm{mperial}$ College, London, UK; ${ }^{2}$ Royal Brompton and Harefield NHS Foundation Trust, London, UK

\subsection{6/thoraxjnl-2015-207770.74}

Adult Primary Ciliary Dyskinesia (PCD) has not been well characterised. Patients have varied radiological severity of disease and lung function impairment and limited data is available regarding prognosis. In this retrospective study we describe and characterise a large adult PCD cohort, and identify determinates of disease progression using longitudinal lung function data.

We retrospectively analysed 151 adult patients at a single tertiary centre. Overall mortality was $4.6 \%$ over a 7 -year median follow-up period. Lung function decline was estimated at $0.49 \%$ $\mathrm{FEV}_{1}$ predicted/year. Older age at diagnosis showed moderate negative correlation with $\mathrm{FEV}_{1} \%$ predicted at diagnosis $(\mathrm{r}=$ $0.30 ; \mathrm{p}=0.01)$ and increased Pseudomonas aeruginosa colonisation $(\mathrm{p}<0.01)$ but not longitudinal $\mathrm{FEV}_{1} \%$ predicted $(\beta=$ 0.001; (95\% CI:-0.35,0.35)). Within multivariate mixed models of FEV $_{1}$ adjusting for ciliary ultrastructure, HRCT scoring of severity of bronchial wall dilatation $(p<0.01)$ and extent of bronchiectasis $(p=0.03)$ showed evidence of modifying the decline in $\mathrm{FEV}_{1}$ with age. Lung function decline additionally differed by ciliary ultrastructure $(p=0.04)$ with patients with microtubular defects having the greatest decline.

Our study reveals a large proportion of adult PCD patients are diagnosed late with lower $\mathrm{FEV}_{1}$ and increased $P$. aeruginosa colonisation at diagnosis. Increased disease burden on HRCT and microtubular defects on ciliary ultrastructure predicts progressive lung function decline. This study highlights the need for early diagnosis alongside prospective multi-centre disease-specific trials to confirm triggers for lung function decline and identify potential novel therapeutic strategies.

\section{S69 DEVELOPMENT OF AN IN VITRO ASSAY TO DETECT CHEMICALLY-INDUCED CHANGES IN CILIARY BEAT FREQUENCY}

${ }^{1}$ A Turnbull, ${ }^{1} \mathrm{~A}$ Shoemark, ${ }^{2} \mathrm{H}$ Lund-Palau, ${ }^{2} \mathrm{~A}$ Bush, ${ }^{3} \mathrm{E}$ Alton, ${ }^{1} \mathrm{I}$ Davies. ${ }^{1}$ National Heart and Lung Institute, Imperial College London and Department of Paediatric Respiratory Medicine, Royal Brompton Hospital, London, UK; ${ }^{2}$ Department of Paediatric Respiratory Medicine, Royal Brompton Hospital, London, UK; ${ }^{3}$ National Heart and Lung Institute, Imperial College London and Department of Adult Respiratory Medicine, Royal Brompton Hospital, London, UK

\subsection{6/thoraxjnl-2015-207770.75}

Techniques are well-established to quantify ciliary beat frequency $(\mathrm{CBF})$, which is often reduced in patients with primary ciliary dyskinesia. This project aims to determine the impact of genetic 